\title{
Fuzzy Variable Linear Programming with Fuzzy Technical Coefficients
}

\author{
Sanwar Uddin Ahmad \\ Department of Mathematics, University of Dhaka \\ Dhaka-1000, Bangladesh \\ sanwar@univdhaka.edu \\ Sadhan Kumar Sardar \\ Department of Mathematics, University of Dhaka \\ Dhaka-1000, Bangladesh \\ sadhanmath@yahoo.com
}

\begin{abstract}
Fuzzy linear programming is an application of fuzzy set theory in linear decision making problems and most of these problems are related to linear programming (LP) with fuzzy variables. In this paper, an approximate but convenient method for solving these problems with fuzzy non-negative technical coefficient and without using the ranking functions, is proposed. With the help of numerical examples, the method is illustrated.
\end{abstract}

Keyword:Fuzzy linear programming, Fuzzy variable linear programming, Fuzzy number, Optimal solution, Decomposition method.

\section{Introduction}

Fuzzy set theory has become an important tool in the branch of decision sciences and has been applied successfully to many disciplines such as control theory, management sciences, mathematical modeling and industrial applications. The concept of fuzzy decision was introduced by Bellman and Zadeh (1970), later Tanaka et. al. (1974) first extended the concept as fuzzy linear programming (FLP) problems on general level. A number of researchers have exhibited their interest to solve the FLP problems and proposed several approaches for solving these problems, see Delgado et. al. (1989), Inuiguchi et al. (2003), Ganesan and Veeramani (2006), after Zimmermann (1985) proposed the formulation of FLP. Existing methods can be divided into two groups in general, depending on the fuzziness of decision parameters and decision variables. In the first group, the researchers assumed the decision parameters are fuzzy numbers while the decision variables are crisp ones; see Buckley (1988), Lai and Hwang (1992), Julien (1994), Shaocheng (1994), Liu (2001). This means that in an uncertain environment, a crisp decision is made to meet some decision criteria.

Tanaka and Asai (1984) can be considered as the pioneers for the second group of FLP problems with fuzzy decision variables and crisp decision parameters. They initially proposed a possibilistic LP formulation and applied LP technique to obtain the largest possibility distribution of the decision variables. The concept of comparison of fuzzy numbers Maleki et al. (1996) is another approach for solving fuzzy number linear programming (FNLP) problems. Later the same authors extended the concept for solving LP problems with fuzzy variables Maleki et al. (2000). In effect, most convenient methods are based on the concept of comparison of fuzzy numbers by use of ranking functions, see Maleki (2002), Nehi et al. (2004). Usually in such methods authors define 
a crisp model which is equivalent to the FLP problem and then use optimal solution of the model as the optimal solution of the FLP problem. Nasseri and Ardil (2005) use linear ranking functions for solving FVLP that uses simplex tableau used to solve LP problems in crisp environment. Mahdavi-Amiri and Nasseri (2007) described duality theory for the FVLP problems.

To the best of our knowledge, all the above authors considered either the technological coefficients or the variable as fuzzy, but not both. However in real-life decision making problems, it is usual that coefficients of LP, where human estimation is used are inexact so as the decision that are taken based on this data. Thus it is desirable to consider the technical coefficients as well as the variables as fuzzy quantities. In this paper we have proposed a method, namely decomposition method, recently used by Pandian and Jayalakshmi (2010), to solve integer linear programming problem with fuzzy variables, for solving FVLP with non-negative fuzzy technological coefficients.

\section{Preliminaries}

We need the following definitions and theorems to establish the method which can be found at Zimmermann $(1985,1996)$.

\subsection{Fuzzy numbers}

Definition 2.1. A fuzzy number $a$ is a convex normalized fuzzy set on the real line $R$ such that:

1) There exists at least one $x_{0} \in R$ with $\mu_{\bar{a}}\left(x_{0}\right)=1$.

2) $\mu_{\bar{a}} \mathrm{x}$ is piecewise continuous.

The membership function of any fuzzy number $a$ is as follows:

$$
\mu_{a} x=\begin{array}{cc}
f x, & x \in a, b \\
1, & x \in b, c \\
g x, & x \in a, b \\
0, & \text { otherwise }
\end{array}
$$

where $a \leq b \leq c \leq d, f$ is increasing and right-continuous function on $[a, b]$, and $g$ is decreasing and left-continuous function on $[b, c]$. If $b b=c c$, then ais a fuzzy number otherwise it is known as fuzzy interval.

Definition 2.2. A fuzzy number $\bar{a}$ is a Triangular Fuzzy Number (TFN) denoted by $a_{1}, a_{2}, a_{3}$ where $a_{1}, a_{2}$ and $a_{3}$ are real numbers and its membership function $\mu_{a} x$ is given below.

$$
\begin{gathered}
\frac{\mathrm{x}-a_{1}}{a_{2}-a_{1}} ; a_{1} \leq x \leq a_{2} \\
\mu_{a} x=\frac{a_{3}-\mathrm{x}}{a_{3}-a_{2}} ; a_{2} \leq x \leq a_{3} \\
\text { Zero; Otherwise }
\end{gathered}
$$


Definition 2.3. A fuzzy number $\left.a=x, \mu_{a} x \quad x \in R\right\}$ is non-negative and denoted by $a \geq 0$ if and only if $\mu_{a} x=0$ for all $x<0$. Then a TFN is non-negative $a=$ $\left(a_{1}, a_{2}, a_{3}\right)$ if and only if $a_{1} \geq 0$.

Definition 2.4. A fuzzy matrix $A=\left[a_{i j}\right]_{m \times n}$ is called non-negative if $a_{i j} \geq 0$, for all $i, j$, where $a_{i j}$ 's are fuzzy numbers.

\subsection{Arithmetic on Fuzzy Numbers.}

Definition 2.5. Let $a$ and $b$ be any fuzzy numbers and let $*$ denote any of the four basic arithmetic operations. Then we define a fuzzy set on $\mathbb{R} \mathbb{R}, a A * b B$ by defining its $\alpha \alpha$ cuts, $\alpha_{a A * b B}=\alpha_{a A B} * \alpha_{b B}$ for any $\alpha \alpha \in(0,1]$. When $*=/$, clearly we have to require that $0 \notin \alpha_{b B}$ for all $\alpha \alpha \in(0,1]$.

Since $\alpha_{a A * b B}$ is a closed interval for each $\alpha \in(0,1]$ and $a, b$ are fuzzy numbers, $a A * b B$ is also fuzzy number, which followed by the following theorem.

Theorem 2.1. Let $* \in\{+,-, \times, /\}$, and let $a, b$ denote any continuous fuzzy numbers. Then the fuzzy set $a A * b B$ defined by

is a continuous fuzzy number.

$$
a A * b B \quad z z=\sup _{\mathrm{z}=\mathrm{x} * \mathrm{y}} \min \left[\mu_{a} x * \mu_{b} y\right]
$$

Proof. Klir and Yuan (1995).

Definition 2.6. Let $a=\left(a_{1}, a_{2}, a_{3}\right)$ and $b=\left(b_{1}, b_{2}, b_{3}\right)$ be two TFNs.

Then

1) $a+b=a_{1}, a_{2}, a_{3}+b_{1}, b_{2}, b_{3}=\left(a_{1}+b_{1}, a_{2}+b_{2}, a_{3}+b_{3}\right)$

2) $a-b=a_{1}, a_{2}, a_{3}-b_{1}, b_{2}, b_{3}=\left(a_{1}-b_{1}, a_{2}-b_{2}, a_{3}-b_{3}\right)$

3) a) $k a=k a_{1}, a_{2}, a_{3}=k a_{1}, k a_{2}, k a_{3}$ fork $\geq 0$

b) $k a=k a_{1}, a_{2}, a_{3}=k a_{3}, k a_{2}, k a_{1}$ fork $\leq 0$

4) $a \cdot b=a_{1}, a_{2}, a_{3} \cdot b_{1}, b_{2}, b_{3}=a_{1} \cdot b_{1}, a_{2} \cdot b_{2}, a_{3} \cdot b_{3}, a, b$ are non-negative TFN's.

Let $F(R)$ be the set of all real TFNs.

Definition 2.7. Let $A=\left(a_{1}, a_{2}, a_{3}\right)$ and $B=\left(b_{1}, b_{2}, b_{3}\right)$ then

I. $A=B \Leftrightarrow a_{i}=b_{i} \forall i=1$ to 3

II. $A \leq B \Leftrightarrow a_{i} \leq b_{i} \forall i=1$ to 3

\section{FVLP Problem with Fuzzy Non-Negative Technical Coefficients}

Consider the following $m \times n$ fuzzy linear system with non-negative real TFNs:

$$
A x \leq b
$$


where the coefficient matrix $A=a_{i j}{ }_{m \times n}=\left(a_{i j}{ }^{1}, a_{i j}{ }^{2}, a_{i j}{ }^{3}\right)_{m \times n}$ is a nonnegative fuzzy matrix with $a_{i j}=\left(a_{i j}{ }^{1}, a_{i j}{ }^{2}, a_{i j}{ }^{3}\right)$ are TFN's and $x=\left(x^{1}, x^{2}, x^{3}\right)_{1 \times m}$, $b=\left(b^{1}, b^{2}, b^{3}\right)_{1 \times m}$ are nonnegative fuzzy vectors and $\mathrm{x}_{\mathrm{j}}, \mathrm{b}_{\mathrm{i}} \in F(R)$, for all $1 \leq j \leq n$ and $1 \leq i \leq m$.

Definition 3.1. A nonnegative fuzzy vector $x$ is said to be a solution of the fuzzy linear system (1) if $x$ satisfies equation (1).

Consider the following FVLP problem

$$
\begin{array}{lr}
\text { Maximize } & z=c x \\
\text { Subject to } & A x \leq b \\
& x \geq 0
\end{array}
$$

where the coefficient matrix $A=a_{i j}{ }_{m \times n}=\left(a_{i j}{ }^{1}, a_{i j}{ }^{2}, a_{i j}{ }^{3}\right)_{m \times n}$ is a nonnegative fuzzy matrix and $x=\left(x^{1}, x^{2}, x^{3}\right)_{1 \times m}, b=\left(b^{1}, b^{2}, b^{3}\right)_{1 \times m}$ are nonnegative fuzzy vectors as defined in (1). If all the above parameters are crisp numbers then (2) is known as the crisp LP problem.

Since support and core of $x_{j}$ is unknown, computing $a_{i j} * x_{j}$ using fuzzy arithmetic is almost impossible. Hence for simplicity in this paper we have considered $\left(a_{i j} * x_{j}\right)$ as TFN, with supports and cores obtained by definition 2.6.

Definition 3.2. A fuzzy vector $x$ is said to be a feasible solution of the problem $(\mathrm{P})$ if $x$ satisfies (2).

Definition 3.3.A feasible solution $x$ of the problem (P) is said to be an optimal solution of the problem (P) if there exists no feasible $u=\left(u_{J}\right)_{n \times 1}$ of $(\mathrm{P})$ such that $c u \geq c x$.

To establish the method we need the following result.

Theorem 3.1. A fuzzy vector $x^{\circ}=\left(x_{1}^{\circ}, x_{2}^{\circ}, x_{3}^{\circ}\right)$ is an approximate optimal solution of the problem (2) if $x_{2}^{\circ}, x_{1}^{\circ}$ and $x_{3}^{\circ}$ are optimal solutions of the following crisp LP problems (AP2), (AP1) and (AP3) respectively where

$$
\begin{array}{ll}
\text { Maximize } & z_{2}=c x_{2} \\
\text { subject to } & A^{2} x_{2} \leq b_{2}
\end{array}
$$

(AP1)

$$
\begin{array}{ll}
\text { Maximize } & z_{1}=c x_{1} \\
\text { subject to } & A^{1} x_{1} \leq b_{1}
\end{array}
$$

$$
x_{2} \geq 0
$$

where $A^{k}=a_{i j}^{k}, \quad k=1,2,3$.

$$
\begin{array}{ll}
\text { Maximize } & z_{3}=c x_{3} \\
\text { subject to } & A^{3} x_{3} \leq b_{3} \\
& x_{3} \geq x_{2}^{\circ}
\end{array}
$$

$$
\begin{aligned}
& x_{1} \leq x_{2}^{\circ} \\
& x_{1} \geq 0
\end{aligned}
$$

$\begin{array}{lll}\text { and (AP3) } & \text { Maximize } & z_{3}=c x_{3} \\ & \text { subject to } & A^{3} x_{3} \leq b_{3} \\ & x_{3} \geq x_{2}^{\circ}\end{array}$

$$
x_{3} \geq 0
$$




\section{Proof:}

The FVLP problem (2) can be written in the following form.

(P) Maximize $\left(z^{1}, z^{2}, z^{3}\right)=c\left(x^{1}, x^{2}, x^{3}\right)$

Subject to $\left(A^{1}, A^{2}, A^{3}\right)\left(x^{1}, x^{2}, x^{3}\right) \leq\left(b^{1}, b^{2}, b^{3}\right)$

$$
x^{1}, x^{2}, x^{3} \geq 0, a_{i j}{ }^{1}, a_{i j}{ }^{2}, a_{i j}{ }^{3} \geq 0
$$

With our assumption about the multiplication of two fuzzy number the above problem approximated to the following problem.

(AP) Maximize $\left(z^{1}, z^{2}, z^{3}\right)=c\left(x^{1}, x^{2}, x^{3}\right)$

Subject to $\left(A^{1} x^{1}, A^{2} x^{2}, A^{3} x^{3}\right) \leq\left(b^{1}, b^{2}, b^{3}\right)$

$$
x^{1}, x^{2}, x^{3} \geq 0, A^{1}, A^{2}, A^{3} \geq 0
$$

Let $x^{\circ}=\left(x_{1}^{\circ}, x_{2}^{\circ}, x_{3}^{\circ}\right)$ be an optimal solution of the problem (AP) and $x=$ $\left(x_{1}, x_{2}, x_{3}\right)$ be a feasible solution of the problem $(\mathrm{P})$. This implies that

$$
\begin{array}{r}
c \mathrm{x}_{1} \leq c \mathrm{x}_{1} ; c x_{2} \leq c x_{2} ; c x_{3} \leq c x_{3} \\
\mathrm{~A}^{1} \mathrm{x}_{1} \leq b_{1} ; A^{2} x_{2} \leq b_{2} ; A^{3} x_{3} \leq b_{3} ; x_{1}, x_{2}, x_{3} \geq 0 \\
\operatorname{Max} z_{1}=c x_{1} ; \operatorname{Max} . z_{2}=c x_{2} ; \operatorname{Max} . z_{3}=c x_{3}
\end{array}
$$

Now, from (3) and (4), we can conclude that $x_{2}^{\circ}, x_{1}^{\circ}$ and $x_{3}^{\circ}$ are optimal solutions of the crisp LP problems (AP2), (AP1) and (AP3).

Suppose that $x_{2}^{\circ}, x_{1}^{\circ}$ and $x_{3}^{\circ}$ are optimal solutions of the crisp LP problems (AP2), (AP1) and (AP3) with optimal values $z_{2}^{\circ}, z_{1}^{\circ}, z_{3}^{\circ}$ respectively. This implies that $x^{\circ}=\left(x_{1}^{\circ}, x_{2}^{\circ}, x_{3}^{\circ}\right)$ is an optimal solution of the problem (AP) with optimal value $z^{\circ}=\left(z_{1}^{\circ}, z_{2}^{\circ}, z_{3}^{\circ}\right)$.

And since (AP) is approximately equivalent to $(\mathrm{P})$, therefore $x^{\circ}=\left(x_{1}^{\circ}, x_{2}^{\circ}, x_{3}^{\circ}\right)$ is an approximate optimal solution of the problem $(\mathrm{P})$ and hence the result.

\section{Algorithm}

Consider the FVLP problem (2).

Step 1.Construct crisp LP problem

$$
\begin{array}{ll}
\text { Maximize } & z^{2}=c x^{2} \\
\text { Subject to } & A^{2} x^{2} \leq b^{2} \\
& x^{2} \geq 0
\end{array}
$$

and let $\mathrm{x}^{2^{\circ}}$ be optimal solution of the problem $\mathrm{P} 2$ 
Step 2. Construct crisp LP problem (AP1)

$$
\begin{aligned}
& \text { Maximize } \quad z^{1}=c x^{1} \\
& \text { Subject to } \quad A^{1} x^{1} \leq b^{1} \\
& x^{2^{\circ}}-x^{1} \geq 0 \\
& x^{1} \geq 0
\end{aligned}
$$

and let $\mathrm{x}^{1^{\circ}}$ be optimal solution of the problem P1

Step 3. Construct crisp LP problem

$$
\begin{array}{ll}
\text { Maximize } & z^{3}=c x^{3} \\
\text { Subject to } & A^{3} x^{3} \leq b^{3} \\
& x^{3}-x^{2^{\circ}} \geq 0 \\
& x^{3} \geq 0
\end{array}
$$

and let $\mathrm{x}^{3^{\circ}}$ be optimal solution of the problem $\mathrm{P} 3$

Then the optimal solution of the original FVLP problem is $x^{\circ}=\left(x^{1^{\circ}}, x^{2^{\circ}}, x^{3^{\circ}}\right)$.

\section{Numerical Example}

To illustrate this method two numerical examples are presented.

\subsection{Example 1}

$$
\begin{aligned}
& P: \operatorname{Max} \quad 8 x_{1}+12 x_{2} \\
& s \quad t 13 x_{1}+16 x_{2} \leq 325 \\
& 10 x_{1}+13 x_{2} \leq 520 \\
& x_{1} \geq 0, x_{2} \geq 0
\end{aligned}
$$

where $x_{1}=x_{1}^{1}, x_{1}^{2}, x_{1}^{2}, x_{2}=x_{2}^{1}, x_{2}^{2}, x_{2}^{2}, 13=10,13,15,16=13,16,20$,

$$
\begin{gathered}
325=200,325,480,10=8,10,13,31=28,31,37,520=(350,520,735) \\
P 2: M a x Z^{2}=8 x_{1}^{2}+12 x_{2}^{2} \\
S \quad t 13 x_{1}^{2}+16 x_{2}^{2} \leq 325 \\
10 x_{1}^{2}+13 x_{2}^{2} \leq 520 \\
x_{2}^{2} \geq 0, x_{2}^{2} \geq 0
\end{gathered}
$$

The optimal solution of the above crisp LP problem is

Step 2:

$$
z^{2}=\frac{2080}{9} \approx 231.11, x_{1}^{2}=\frac{65}{9} \approx 7.22, x_{2}^{2}=\frac{130}{9} \approx 14.44
$$

$$
\begin{gathered}
P 1: \operatorname{Maxz}^{1}=8 x_{1}^{1}+12 x_{2}^{1} \\
{ }_{t} 10 x_{1}^{1}+13 x_{2}^{1} \leq 200 \\
8 x_{1}^{1}+28 x_{2}^{1} \leq 350 \\
x_{1}^{1} \leq \frac{65}{9} \\
x_{2}^{1} \leq \frac{130}{9} \\
x_{2}^{1} \geq 0, x_{2}^{1} \geq 0
\end{gathered}
$$


The optimal solution of the above crisp solution is

Step 3:

$$
z^{1}=\frac{1950}{11} \approx 177.27, \mathrm{x}_{1}^{1}=\frac{525}{88} \approx 5.96, \mathrm{x}_{2}^{1}=\frac{475}{44} \approx 10.79
$$

$$
\begin{aligned}
& \text { P3: } \operatorname{Maxz}^{3}=8 x_{1}^{3}+12 x_{2}^{3} \\
& { }^{s}{ }_{t} 15 x_{1}^{3}+20 x_{2}^{3} \leq 480 \\
& 13 x_{1}^{3}+37 x_{2}^{3} \leq 735 \\
& x_{1}^{3} \geq \frac{65}{9} \\
& x_{2}^{3} \geq \frac{130}{9} \\
& x_{2}^{3} \geq 0, x_{2}^{3} \geq 0
\end{aligned}
$$

The optimal solution of the above crisp solution is

$$
z^{3}=\frac{16380}{59} \approx 277.62, \mathrm{x}_{1}^{3}=\frac{612}{59} \approx 10.37, \mathrm{x}_{2}^{3}=\frac{957}{59} \approx 16.22 .
$$

Then the optimal solution of the original FVLP problem $\mathrm{P}$ is

$\mathrm{x}_{1}=\frac{525}{88}, \frac{65}{9}, \frac{612}{59} \approx 5.96,7.22,10.37, \mathrm{x}_{2}=\frac{475}{44}, \frac{130}{9}, \frac{957}{59} \approx 10.79,14.44,14.22$ and $\mathrm{z}=\frac{1950}{11}, \frac{2080}{9}, \frac{16380}{59} \approx(177.27,231.11,277.62)$.

\subsection{Example 2}

$$
\begin{aligned}
& P: \operatorname{Max} 5 x_{1}+8 x_{2} \\
& S \quad 9 x_{1}+7 x_{2} \leq 117 \\
& t x_{1}+17 x_{2} \leq 207 \\
& x_{1} \geq 0, x_{2} \geq 0
\end{aligned}
$$

where $x_{1}=x_{1}^{1}, x_{1}^{2}, x_{1}^{2}, x_{2}=x_{2}^{1}, x_{2}^{2}, x_{2}^{2}, 9=5,9,15,7=4,7,10,117=$

$$
7=4,7,13,17=14,17,21,207=(100,207,420)
$$

P2.

$$
\begin{aligned}
& \operatorname{Maxz}^{2}=5 x_{1}^{2}+8 x_{2}^{2} \\
& { }^{s}{ }_{t} 9 x_{1}^{2}+7 x_{2}^{2} \leq 117 \\
& 7 x_{1}^{2}+17 x_{2}^{2} \leq 207 \\
& x_{2}^{2} \geq 0, x_{2}^{2} \geq 0
\end{aligned}
$$

The optimal solution of the above crisp LP problem is

Step 2:

$$
z^{2}=\frac{2763}{26} \approx 106.27, x_{1}^{2}=\frac{135}{26} \approx 5.19, x_{2}^{2}=\frac{261}{26} \approx 10.04
$$

$$
\begin{aligned}
& P 1: \operatorname{Maxz}^{1}=5 x_{1}^{1}+8 x_{2}^{1} \\
& { }^{s}{ }_{t} 5 x_{1}^{1}+4 x_{2}^{1} \leq 40 \\
& 4 x_{1}^{1}+14 x_{2}^{1} \leq 100 \\
& x_{1}^{1} \leq \frac{135}{26} \\
& x_{2}^{1} \leq \frac{261}{26} \\
& x_{2}^{1} \geq 0, x_{2}^{1} \geq 0
\end{aligned}
$$


The optimal solution of the above crisp solution is

Step 3:

$$
Z^{1}=\frac{1760}{27} \approx 65.19, \mathrm{x}_{1}^{1}=\frac{80}{27} \approx 2.96, \mathrm{x}_{2}^{1}=\frac{170}{27} \approx 6.39 .
$$

$$
\begin{gathered}
P 3: \operatorname{Max}^{3}=5 x_{1}^{3}+8 x_{2}^{3} \\
s \quad 10 x_{1}^{3}+15 x_{2}^{3} \leq 270 \\
13 x_{1}^{3}+21 x_{2}^{3} \leq 420 \\
x_{1}^{3} \geq \frac{135}{26} \\
x_{2}^{3} \geq \frac{261}{26} \\
x_{2}^{3} \geq 0, x_{2}^{3} \geq 0
\end{gathered}
$$

The optimal solution of the above crisp solution is

$$
z^{3}=\frac{5934}{37} \approx 160.38, \mathrm{x}_{1}^{3}=\frac{294}{37} \approx 7.94, \mathrm{x}_{2}^{3}=\frac{558}{37} \approx 15.08
$$

Then the optimal solution of the original FVLP problem $\mathrm{P}$ is

$\mathrm{x}_{1}=\frac{80}{27}, \frac{135}{26}, \frac{294}{37} \approx 2.96,5.19,7.94, \mathrm{x}_{2}=\frac{80}{27}, \frac{135}{26}, \frac{558}{37} \approx 6.39,10.04,15.08$ and $\mathrm{z}=\frac{1760}{27}, \frac{2763}{26}, \frac{5934}{37} \approx(65.19,106.27,160.38)$.

\section{Conclusion}

In this paper FVLP problems with non-negative Fuzzy Technical coefficients are considered. A new approach has been provided for solving such problems. The proposed method provides an approximation to the optimal solution without using ranking functions and applying classical LP technique. Method is simple and efficient and can be applied in anywhere. If the fuzzy numbers are crisp, the method produces three crisp LP problems with same optimum solutions.

\section{References}

1. Bellman, R.E. and Zadeh, L.A. (1970). Decision making in a fuzzy environment, Management Sci. 17, 141-164.

2. Buckley, J.J. (1988).Possibilistic linear programming with triangular fuzzy numbers, Fuzzy Sets and Systems 26, 135-138.

3. Delgado, M. and Verdegay, J. L. and Vila, M. A. (1989). A general model for fuzzy linear programming, Fuzzy Sets and Systems, 29, 21-29.

4. Ganesan, K. and Veeramani, P. (2006). Fuzzy linear programming with trapezoidal fuzzy numbers, Ann. Oper. Res. 143, 305-315.

5. Inuiguchi, M., Ramik, J., Tanino, T. and Vlach, M. (2003). Satisficing solutions and duality in interval and fuzzy linear programming, Fuzzy Sets and Systems $135,151-177$.

6. Julien, B. (1994).A extension to possibilistic linear programming, Fuzzy Sets and Systems 64, 195-206. 
7. Klir, G. and Yuan, B. (1995). Fuzzy Set and Fuzzy Logic, Prentice-Hall, Inc. Upper Saddle River, New Jersey 07458, U.S.A.

8. Lai, Y.J. and Hwang, C.L. (1992). A new approach to some possibilistic linear programming problem, Fuzzy Sets and Systems 49, 121-133.

9. Liu, X. (2001). Measuring the satisfaction of constraints in fuzzy linear programming, Fuzzy Sets and Systems 122, 263-275.

10. Mahdavi-Amiri, N. and Nasseri, S.H. (2007). Duality results and a dual simplex method for linear programming problems with trapezoidal fuzzy variables, Fuzzy Sets and Systems 158, 1961 - 1978.

11. Maleki, H.R., Tata, M., Mashinchi, M. (1996). Fuzzy number linear programming, in: C. Lucas (Ed.), Proc. Internat. Conf. on Intelligent and Cognitive Systems FSS '96, sponsored by IEE and ISRF, Tehran, Iran, pp. 145148.

12. Maleki, H.R., Tata, M., Mashinchi, M. (2000). Linear Programming with fuzzy variables, Fuzzy Sets and Systems, 109, 21-33.

13. Maleki, H.R. (2002). Ranking functions and their applications to fuzzy linear programming, Far East J. Math. Sci. (FJMS) 4, 283-301.

14. Nehi, H.M., Maleki, H.R., Mashinchi, M. (2004). Solving fuzzy number linear programming problem by Lexicographic ranking function, Italian J. Pure Appl. Math. 15, 9-20.

15. Nasseri, S.H. and Ardil, E., (2005). Simplex Method for Fuzzy Variable Linear Programming Problems, World Academy of Science, Engineering and Technology 8, 198-202.

16. Pandian, P. and Jayalakshmi, M., (2010). A New Method for Solving Integer Linear Programming Problems with Fuzzy Variables, Applied Mathematical Sciences, Vol. 4, no. 20, 997 - 1004.

17. Shaocheng, T., (1994). Interval number and fuzzy number linear programming, Fuzzy Sets and Systems 66, 301-306.

18. Tanaka, H., Okuda, T. and Asai, K. (1974). On fuzzy mathematical programming, The Journal of Cybernetics, 3, 37-46.

19. Tanaka, H. and Asai, K. (1984). Fuzzy solution in fuzzy linear programming problems, IEEE Trans. System Man Cybrenet. 14, 325-328.

20. Zimmermann, H.J. (1985). Applications of fuzzy sets theory to mathematical programming, Inform. Sci. 36, 29-58.

21. Zimmermann, H.J. (1996). Fuzzy Set Theory and its application, Kluwer Academic Publishers. 